\title{
¿Es posible disminuir la mortalidad por cáncer de mama en Chile? Análisis epidemiológico y programático en la Región Metropolitana, 2000-2007*
}

\author{
Drs. IVÁN SERRA C. ${ }^{1}$, RAÚl MARTÍNEZ R. ${ }^{1}$, XIMENA MIMICA S. ${ }^{1}$, CLAUDIA AGUAYO S. ${ }^{2}$ \\ Servicio de Cirugía Hospital Sótero del Río, Santiago. \\ 2 Magíster en Salud Pública, enfermera y epidemióloga, Hospital Regional, Concepción. \\ Chile.
}

\section{Abstract}

\section{Is it possible to decrease breast cancer mortality in Chile? An analytical study}

This analytical study is aimed to analyze breast cancer mortality in the metropolitan region of Santiago serving something more than $6,000,000$ people. The hypothesis is that it is possible to decrease breast cancer mortality in Chile (16 million inhabitants) based on the experience of the metropolitan south orient health service (SSMSO) that represents $10 \%$ of the Chilean population. A comparative analysis of crude and ageadjusted mortality rates for breast cancer in the six metropolitan health services is produced showing the lowest crude and age-adjusted mortality rate in the SSMSO besides to be lower than the mortality rate for Chile. Furthermore the annual proportions of advanced (III and IV) and incipient breast cancers (in situ and I) are established for the SSMSO showing a proportional decrease of advanced stages and a relevant increase of the incipient ones between 1994 and 2007. The authors think that since the population of the metropolitan region represents more than $40 \%$ of the Chilean population and includes all the socio economic strata the results observed in the SSMSO, the biggest health service in the region besides having both rural and urban districts, may be considered representative for the country as a whole. The original hypothesis has been thus confirmed: it is possible to decrease breast cancer mortality in Chile.

Key words: Breast cancer, epidemiology, mortality, health programs, Chile.

\section{Resumen}

Se trata un estudio analítico sobre la mortalidad por cáncer de mama en la Región Metropolitana. La hipótesis, en base a la experiencia del Servicio de Salud Metropolitano Sur Oriente (SSMSO), señala que es posible disminuir la mortalidad por este cáncer en Chile, extrapolando la experiencia de este servicio de salud con 1,6 millones de población asignada y 1,1 millones de personas inscritas validadas. Ello significa el $10 \%$ de la población chilena. Se hace un análisis comparado de las mortalidades crudas y ajustadas por edad en los seis servicios de salud metropolitanos, período 2000-2007, además de establecer la proporción anual de cánceres de mama avanzados (III y IV) e incipientes (in situ y I) en el total de cánceres mamarios del SSMSO, período 1994-2007. Los seis servicios en conjunto atienden una población que significa algo más del

\footnotetext{
*Recibido el 10 de Diciembre de 2009 y aceptado para publicación el 23 de Febrero de 2010.

Correspondencia: Dr. Iván Serra C.

Concha y Toro 3459. Puente Alto, Santiago, Chile.

E-mail: ivan.serra.canales@gmail.com
} 
$40 \%$ de la nacional, además de representar todos sus estratos socio-económicos y étnicos. En consecuencia, la comparación entre ellos puede considerarse válida para el país. Los resultados muestran que el SSMSO tiene la mortalidad por cáncer de mama, tasa cruda y ajustada, más baja de la Región Metropolitana, además de inferior a la nacional. Una razón importante es la disminución proporcional de cánceres avanzados y un aumento importante de los incipientes. El programa para cáncer de mama del SSMSO cumple entonces con su objetivo principal: disminuir la mortalidad por este cáncer, ratificando la hipótesis de este estudio. Además, cumple otros objetivos: eficiencia, buena opinión de usuarias y equidad.

Palabras clave: Cáncer de mama, epidemiología, mortalidad, programas de salud, Chile.

\section{Introducción}

Sin duda, el cáncer de mama preocupa a la sociedad chilena. Sin embargo, esta inquietud pareciera dirigirse en nuestro sistema de salud, de preferencia al diagnóstico y en especial al tratamiento, sin considerar debidamente lo preventivo, epidemiológico y programático.

Los países desarrollados han enfrentado por más de 100 años esta muy frecuente enfermedad en sus poblaciones y la siguen enfrentando con mucha fuerza y recursos, aportando al mundo una enorme cantidad de investigaciones, conocimiento científico y experiencias clínicas $^{1-6}$. En Chile, al existir una frecuencia mucho menor deberíamos aprovechar no sólo las grandes casuísticas extranjeras incluyendo tecnologías modernas como quimio-sensibilidad ${ }^{7-9}$ sino también sus aportes en lo preventivo. Pero, además, es imperativo aportar nuestras experiencias clínicas y epidemiológicas, incluyendo lo analítico y elementos factibles de introducir para potenciar nuestros programas ${ }^{10-16}$.

El objetivo principal del programa nacional de cáncer de mama en Chile, en desarrollo desde 1995, es disminuir la mortalidad por esta causa lo cual no se ha cumplido ${ }^{17}$. Su no cumplimiento impulsa a conocer las razones que lo explicarían y a explorar estrategias que faciliten cumplir dicho objetivo. Por ello, es útil destacar que un programa de salud pública debe contemplar cuatro objetivos importantes ${ }^{18}$.

El principal objetivo es obtener un impacto sanitario, o sea, evitar el desarrollo de una patología determinada y rebajar así su incidencia real, no sólo la captación de nuevos casos o incidencia registrada. Esto corresponde a la prevención primaria. En cáncer de mama, una neoplasia relacionada con los estrógenos, existen alternativas efectivas para disminuir el riesgo de desarrollar la enfermedad ${ }^{19-24}$.

Si ello no es factible, el programa deberá dirigirse a disminuir la mortalidad en los casos que se presentan. En cáncer de mama, es posible a través de un diagnóstico en etapas incipientes, precoces o tempranas verdaderas, lo cual se puede lograr por tamizaje, especialmente con mamografía ${ }^{25-27}$. Es la prevención secundaria. Además, a través de educación del personal de salud y de la población que incluya auto-examen mamario ${ }^{28,29}$, aunque la utilidad de esto último está actualmente en debate.

Debe existir también una buena gestión del programa. Ello comprende mejor captación de nuevas pacientes, afinamiento del proceso de diagnóstico y tratamiento junto con una evaluación integrada entre los diferentes niveles de decisión: central, regional y local, además de los tres niveles de atención: primario, secundario y terciario. También, los tratamientos deben ser oportunos, eficaces y con evaluación permanente.

Un segundo objetivo es la eficiencia: máxima efectividad con los menores costos posibles. Por ello deben priorizarse los aspectos preventivos, basando el programa en un buen diagnóstico epidemiológico que considere la incidencia local, no las correspondientes a países desarrollados con magnitudes varias veces superiores a la nuestra.

Un tercer objetivo es obtener buena opinión de las usuarias ${ }^{30}$. Es fundamental en esta patología tener una oportuna captación de pacientes que incluya idealmente el estudio y seguimiento de las hijas adultas de mujeres atendidas con cáncer de mama.

Un cuarto objetivo es promover la equidad, no como concepto teórico sino en la atención de salud, o sea, centrado en las personas con mayores necesidades $^{31}$.

El objetivo de nuestro estudio es evaluar el programa para cáncer de mama desarrollado durante dos décadas en nuestro Servicio de Salud Metropolitano Sur Oriente (SSMSO), comparando su mortalidad con los otros cinco de servicios de salud metropolitanos, período 2000-2007. La hipótesis es que, sin disponer de programas de tamizaje y atendiendo una población predominantemente de estratos socioeconómicos medio-bajos y bajos, ha sido posible reducir en el SSMSO la mortalidad por cáncer de mama, haciéndolo además en forma eficiente, con buena opinión de usuarias y privilegiando la equidad, además de mostrar éxito en un plazo razonablemente corto con un aumento de la incidencia global, un descenso de la tasa de cánceres avanzados y un incremento importante de los incipientes. 


\section{Material y Método}

Con el objeto de comparar la situación epidemiológica y programática de los seis servicios de salud metropolitanos, período 2000-2007, se calcularon las tasas crudas de mortalidad en estos seis servicios, con datos del departamento de estadísticas e información en salud (DEIS), Ministerio de Salud y la base poblacional del Instituto Nacional de Estadística (INE), estableciendo promedios anuales para cada servicio en todo el período y en cada año. Se eligió el 2006 para efectuar un ajuste por edad por ser el año con el promedio más cercano al promedio del período 2000-2007 de los seis servicios de salud (excepto el 2000 que se consideró muy antiguo).

Se estableció la proporción de cánceres avanzados (III y IV) e incipientes (in situ y I) del SSMSO, 1994-2007, para relacionar su evolución con la equivalente en mortalidad 2000-2007.

Para conocer la eficiencia se asumieron los gastos del programa de cáncer de mama en el SSMSO en base al número y costo de mamografías, ecografías mamarias, biopsias, intervenciones quirúrgicas (valores programa oportunidad de la atención, POA), radioterapias (convenios) y quimioterapias (gasto de farmacia) en todos los pacientes 2000-2007.

La opinión de usuarias se estableció en base al cumplimiento de indicaciones, adherencia al tratamiento, expresiones recibidas en los controles y talleres educativos, seguimiento telefónico y domiciliario, en una evaluación cualitativa.

La equidad se midió según proporción de pacientes del Fondo Nacional de Salud (Fonasa) grupos A y B, comparado con grupos C y D o eventualmente, con otro tipo de seguro en salud.

\section{Resultados}

Se presentan las tasas crudas de mortalidad por cáncer de mama para cada servicio de salud y año.
Además, se presenta el gran total que corresponde al promedio de estos dos promedios, todo lo cual permite apreciar situaciones diferentes según el servicio y año considerado (Tabla 1).

El ajuste por edad de esas tasas en los seis servicios de salud metropolitanos muestra algunas variaciones que no aparecen como relevantes. Primero, la gran diferencia entre los dos extremos se atenúa por disminución en el servicio con mayor mortalidad, oriente, por su población más envejecida y aumenta en el de menor mortalidad, sur oriente, dada su población más joven. Sin embargo, persiste una gran diferencia entre estos dos servicios con un diferencial que supera los cuatro puntos el año 2006. Segundo, el SSMSO es el único de los seis servicios con una mortalidad ajustada por edad bajo el promedio nacional. El servicio occidente muestra una mortalidad cercana a la del oriente, también con alta mortalidad (en este año particular que no coincide con lo observado en otros años), mientras los otros tres servicios se agrupan en un nivel intermedio, también por encima del promedio nacional (Figura 1).

El análisis de las tasas crudas de mortalidad como curvas, 2000-2007, en los seis servicios de salud metropolitanos, muestra evoluciones y niveles de mortalidad interesantes en su comparación. El Servicio Oriente aparece con la más alta mortalidad y su alto nivel es además estable. El Sur Oriente en el otro extremo, también presenta una mortalidad estable pero muy baja, la de menor magnitud comparada. Los otros cuatro servicios muestran diferentes tendencias en niveles intermedios entre los dos anteriores. El Servicio Occidente tiende al aumento aunque es el segundo servicio con menor nivel promedio de mortalidad, especialmente el 2007. El servicio Sur también presenta tendencia al aumento, pero con un mayor nivel de mortalidad. El Servicio Central muestra mucha irregularidad con un relativo alto nivel. Finalmente, el Servicio Norte presenta magnitudes bastante variables, en un nivel

Tabla 1. Tasas crudas de mortalidad por cáncer de mama. Servicios de Salud Metropolitanos, 2000-2007*

\begin{tabular}{lccccccccc}
\hline Servicio de Salud & $\mathbf{2 0 0 0}$ & $\mathbf{2 0 0 1}$ & $\mathbf{2 0 0 2}$ & $\mathbf{2 0 0 3}$ & $\mathbf{2 0 0 4}$ & $\mathbf{2 0 0 5}$ & $\mathbf{2 0 0 6}$ & $\mathbf{2 0 0 7}$ & Promedios SS SS** \\
\hline Oriente & 23,9 & 20,9 & 20,5 & 20,3 & 21,9 & 22,6 & 22,6 & 20,2 & 21,5 \\
Sur & 13,2 & 16,3 & 14,6 & 14 & 15,5 & 15,3 & 16,4 & 16,2 & 15,2 \\
Central & 14,8 & 13,4 & 13,7 & 16 & 18,8 & 16,4 & 13,9 & 14,6 & 15,2 \\
Norte & 15,4 & 11,6 & 14,8 & 12,7 & 17,6 & 13,8 & 15,1 & 13,8 & 14,4 \\
Occidente & 11 & 12,5 & 12,8 & 12,9 & 14,7 & 13,5 & 15,4 & 11,9 & 13,1 \\
Sur Oriente & 12,7 & 10,7 & 12,2 & 11,5 & 11,3 & 12,2 & 10,7 & 11,2 & 11,6 \\
Promedios RM & 15,2 & 14,2 & 14,8 & 14,6 & 16,6 & 15,6 & 15,5 & 14,7 & 15,2 \\
\hline
\end{tabular}

*Tasas por 100.000 mujeres. **Servicios de Salud. 


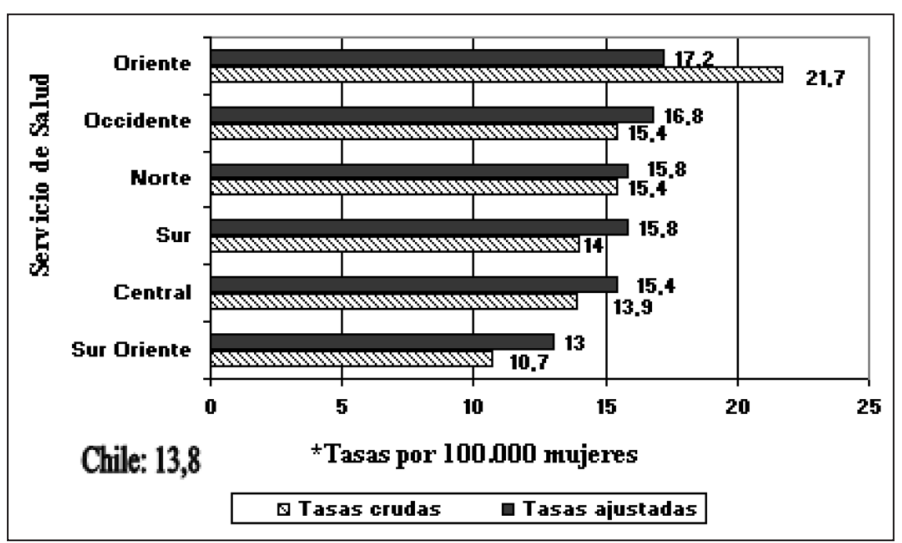

Figura 1. Tasas de mortalidad crudas y ajustadas por edad. Servicios de Salud Metropolitanos, 2006*.

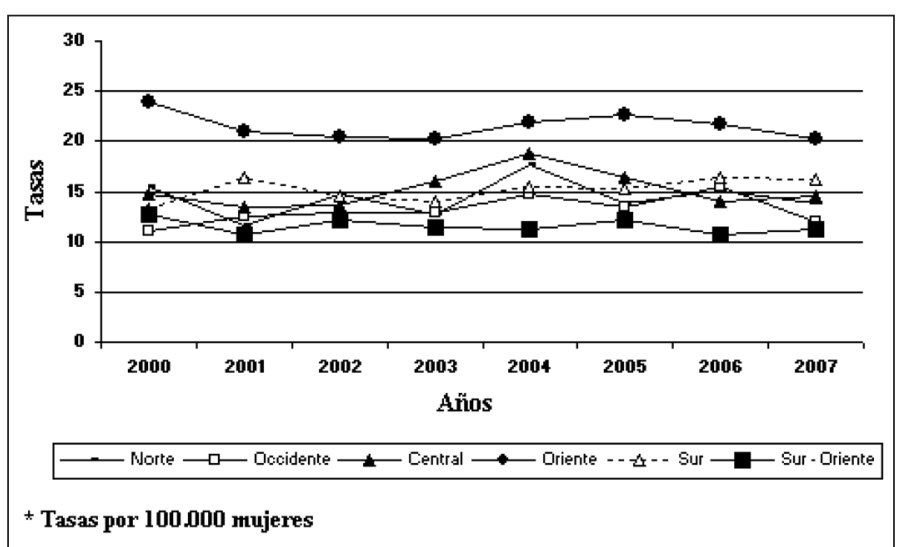

Figura 2. Tasas de mortalidad crudas por cáncer de mama. Servicios de Salud Metropolitanos, 2000-2007*. intermedio. En otras palabras, salvo los servicios oriente y sur oriente con mortalidades estables 2000-2007 aunque a muy diferente nivel, en los otros cuatro su mortalidad por cáncer de mama es variable según el año que se considere (Figura 2).

Datos publicados muestran un nivel de mortalidad por cáncer de mama en el SSMSO del orden de 12 y 13 por 100.000 mujeres en la década del 90 , mortalidad que ha bajado en la actualidad a 11 y menos ${ }^{16}$. Esto se correlaciona con una proporción decreciente de cánceres avanzados (III y IV), los cuales habían aumentado en los 90 hasta sobrepasar el 50\% del total de cánceres mamarios para disminuir después en forma progresiva hasta alcanzar $21 \mathrm{y}$ $22 \%$ en los últimos años. Esta proporción está por debajo del nivel nacional que sería de $30 \%{ }^{17}$. En relación a los incipientes (in situ y I), su proporción en el SSMSO ha aumentado en forma sostenida en la década actual hasta alcanzar 34\% el 2007 (Figuras 3 y 4).

El gasto del programa en el SSMSO es relativamente bajo. El número de exámenes diagnósticos institucionales en el período 2000-2007 es bastante escaso en general, aunque su registro no incluye los exámenes efectuados por cuenta de las pacientes. Esto se observa especialmente hasta el año 2003 para mamografías y hasta el 2005 para ecografías mamarias. En mamografías

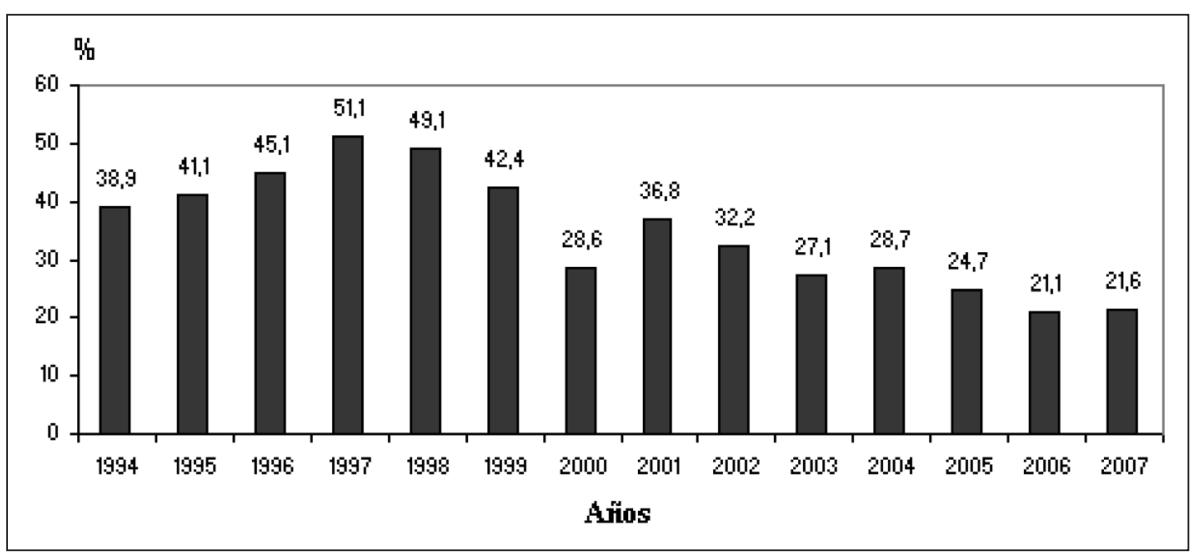

Figura 3. Proporción anual de cánceres de mama avanzados etapa III y IV. SSMO 1994-2007. 
existe un gran aumento de los exámenes efectuados a nivel municipal con financiamiento ministerial sólo el año 2007. El costo por mamografía, institucional o municipalizada, fue inferior a $\$ 15.000$, mientras para las ecografías fue algo más bajo, inferior a \$12.000. Las biopsias estereotáxicas, efectuadas todas en un establecimiento externo, con un promedio cercano a 70 procedimientos anuales de los cuales unos 20 correspondieron a cáncer de mama, tuvieron un alto costo unitario de $\$ 620.000$.

En lo terapéutico, el gasto es proporcional al aumento de la incidencia ya que en la actualidad se atienden casi 300 pacientes anuales comparado con menos de 200 entre el 2000 y el 2005. Los tratamientos conservadores (mastectomías parciales) aumentaron desde 40 el año 2000 hasta casi 200 el 2007, mientras las cirugías radicales (mastectomías totales) aumentaron desde 30-56 a comienzos de la década del 2000 hasta 74-107 en la actualidad. Esta mejoría en la conservación mamaria responde en gran medida a la disminución de cánceres avanzados e incremento de incipientes. La razón mastectomías parciales/mastectomías totales fue el año 2000 de 0,7:1 mejorando progresivamente hasta ser 1,5:1 el año 2007. Por lo tanto, se ha producido una inversión completa de la relación entre ambos tipos de cirugía mamaria en un período de sólo ocho años. El costo de las mastectomías, arancel POA, ascendió a $\$ 300.000$ y $\$ 400.000$ según el tipo de intervención. Las linfocintigrafías con inyección de un radiofármaco para intervenciones de linfonodo centinela, se realizaron en un establecimiento externo, con un número de 30 anuales en promedio, lo que significó un costo unitario de $\$ 300.000$. Las radioterapias, respaldadas por dos convenios, fueron unas 100 anuales entre el 2000 y el 2003, subiendo a casi 200 anuales en los últimos años, con un gasto de $\$ 420.000$ y $\$ 490.000$ según el convenio usado (Tabla 2).

El tercer objetivo del programa: satisfacción de usuarias, fue establecido en forma cualitativa a través de la ausencia de críticas y a una buena cantidad de felicitaciones recibidas en las atenciones realizadas. Igualmente, en los cientos de llamados telefónicos y decenas de visitas domiciliarias practicadas. No es casualidad un seguimiento de prácticamente el 100\%. En consecuencia, estos hechos muestran una evaluación muy positiva del programa.

La distribución por grupo previsional mostró que la mayor parte de las pacientes fue Fonasa B y una menor proporción, aunque importante estuvo en categoría A, fundamentalmente indigentes. Sólo una proporción baja, 25\%, correspondió a los estratos $\mathrm{C}$ y $\mathrm{D}$ en conjunto. Hubo muy aislados casos, $0,3 \%$ del total de pacientes, adscritos a Instituciones de Salud Previsional (Isapres). Las cifras señaladas incluyen a las pacientes privadas institucionales. Aunque la equidad no fue objetivamente medida en este estudio, no hubo diferencias en la oportunidad del acceso al diagnóstico y al tipo de tratamiento en las pacientes según el grupo previsional del que provenían, por lo que podemos afirmar que la equidad fue otro objetivo cumplido en el SSMSO (Tabla 3).

\section{Discusión}

La principal fortaleza de este estudio es desarrollar por primera vez una evaluación del programa nacional de cáncer de mama que permite aportar elementos para disminuir la mortalidad por esta causa. El SSMSO ha logrado hacerlo, obteniendo la

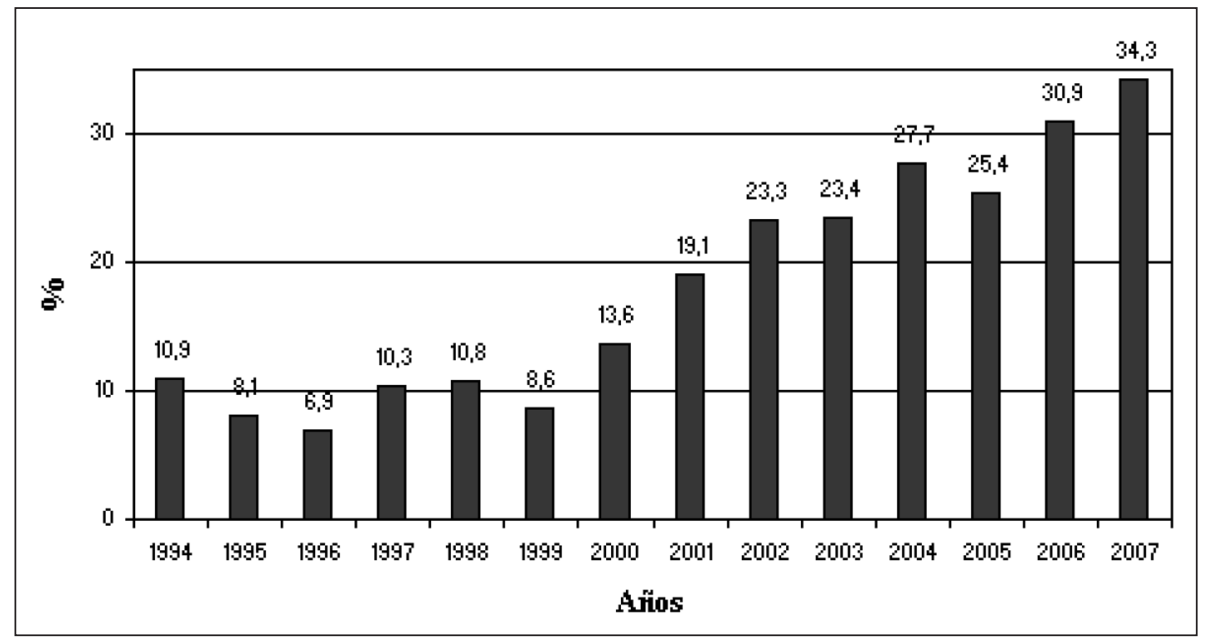

Figura 4. Proporción anual de cánceres de mama incipientes (in situ y I). SSMO 1994-2007. 
Tabla 2. Número y costo de procedimientos diagnósticos y terapéuticos en cáncer de mama. Servicio de Salud Metropolitano Sur Oriente, 2000-2007

\begin{tabular}{|c|c|c|c|c|c|c|c|c|c|c|c|}
\hline \multirow[b]{2}{*}{ Años } & \multirow[b]{2}{*}{$\begin{array}{c}\mathrm{N}^{\circ} \\
\text { pacien- } \\
\text { tes }\end{array}$} & \multicolumn{3}{|c|}{ Mamografías } & \multicolumn{3}{|c|}{ Ecografías } & \multirow{2}{*}{$\begin{array}{l}\text { Total } \\
\text { opera- } \\
\text { ciones }\end{array}$} & \multirow{2}{*}{$\begin{array}{l}\text { Radio- } \\
\text { terapias }\end{array}$} & \multirow{2}{*}{$\begin{array}{l}\text { Quimio- } \\
\text { terapias }\end{array}$} & \multirow{2}{*}{$\begin{array}{c}\text { Hormono- } \\
\text { terapias }\end{array}$} \\
\hline & & Instit & Munic & Total & Instit & Munic & Total & & & & \\
\hline 2000 & 143 & 934 & 0 & 934 & 76 & 0 & 76 & 143 & 95 & 84 & 18 \\
\hline 2001 & 144 & 1.097 & 0 & 1.097 & 0 & 0 & 0 & 144 & 100 & 79 & 52 \\
\hline 2002 & 155 & 1.468 & 0 & 1.468 & 0 & 0 & 0 & 155 & 112 & 128 & 38 \\
\hline 2003 & 184 & 998 & 0 & 998 & 27 & 0 & 27 & 184 & 110 & 111 & 40 \\
\hline 2004 & 202 & 0 & 4.424 & 4.424 & 0 & 0 & 0 & 202 & 145 & 147 & 52 \\
\hline 2005 & 206 & 2.189 & 6.447 & 8.636 & 28 & 0 & 28 & 206 & 147 & 163 & 159 \\
\hline 2006 & 249 & 2.938 & 7.514 & 10.452 & 1.056 & 924 & 1.976 & 249 & 173 & 174 & 192 \\
\hline 2007 & 295 & 5.932 & 14.404 & 20.336 & 1.919 & 3.505 & 5.424 & 295 & 188 & 127 & 130 \\
\hline
\end{tabular}

\begin{tabular}{|c|c|c|}
\hline Costo por mamografía & 13.480 & \\
\hline Costo por ecografía mamaria & 11.340 & \\
\hline Costo biopsia estereotáxica & : \$ 620.000 & \\
\hline \multirow[t]{2}{*}{ Costo por intervención quirúrgica } & : \$ 300.000 & (parcial) \\
\hline & $\$ \quad 400.000$ & (total) \\
\hline Costo por tratamiento de radioterapia & : \$ 420.000 & \\
\hline \multirow[t]{4}{*}{ Costo por tratamiento de quimioterapia } & : \$ 128.000 & (CMF) ciclofosfamida-metotrexato-fluoruracilo \\
\hline & $\$ \quad 160.000$ & (AC) adriamicina-ciclofosfamida \\
\hline & $\$ \quad 270.000$ & (FAC) fluoruracilo-adriamicina-ciclofosfamida \\
\hline & $\$ 2.120 .000$ & (T) taxanos \\
\hline \multirow[t]{2}{*}{ Costo anual por hormonoterapia } & $\$ \quad 16.284$ & tamoxifeno \\
\hline & $\$ 1.103 .760$ & Inhibidores aromatasa \\
\hline
\end{tabular}

Tabla 3. Condición previsional de pacientes con cáncer de mama. Servicio de Salud Metropolitano Sur Oriente 2000-2007

\begin{tabular}{rcrrrrrrrrrrrr}
\hline Años & $\begin{array}{c}\mathbf{N}^{\mathbf{0}} \mathbf{d e} \\
\text { pacientes }\end{array}$ & \multicolumn{2}{c}{$\begin{array}{c}\text { Pacientes } \\
\text { con información }\end{array}$} & \multicolumn{2}{c}{ Fonasa A } & \multicolumn{2}{c}{ Fonasa B } & \multicolumn{2}{c}{ Fonasa C } & \multicolumn{2}{c}{ Fonasa D } & \multicolumn{2}{c}{ Isapre } \\
& & $\mathbf{n}$ & $\mathbf{\%}$ & $\mathbf{n}$ & $\mathbf{\%}$ & $\mathbf{n}$ & $\mathbf{\%}$ & $\mathbf{n}$ & $\mathbf{\%}$ & $\mathbf{n}$ & $\mathbf{\%}$ & $\mathbf{n}$ & $\mathbf{\%}$ \\
\hline 2000 & 143 & 130 & 91,0 & 29 & 22,3 & 75 & 57,7 & 11 & 8,5 & 14 & 10,4 & 1 & 0,8 \\
2001 & 144 & 124 & 86,1 & 39 & 31,5 & 57 & 46,0 & 14 & 11,3 & 14 & 11,3 & - \\
2002 & 155 & 136 & 87,7 & 40 & 29,4 & 62 & 45,6 & 21 & 15,4 & 12 & 5,8 & 1 & 0,7 \\
2003 & 184 & 170 & 92,3 & 54 & 31,8 & 73 & 42,9 & 21 & 12,4 & 21 & 12,4 & 1 & 0,6 \\
2004 & 202 & 198 & 98,0 & 63 & 31,8 & 83 & 47,0 & 22 & 11,1 & 20 & 10,1 & - \\
2005 & 206 & 197 & 95,6 & 63 & 32,0 & 83 & 42,1 & 29 & 14,7 & 22 & 11,2 & - \\
2006 & 249 & 228 & 91,5 & 56 & 24,6 & 106 & 46,5 & 26 & 11,4 & 40 & 17,5 & - \\
2007 & 295 & 275 & 93,2 & 69 & 25,1 & 127 & 42,2 & 33 & 12,0 & 45 & 16,4 & 1 & 0,4 \\
Total & 1.578 & 1.478 & 93,6 & 413 & 283 & 619 & 42,5 & 177 & 12,1 & 188 & 12,9 & 4 & 0,3 \\
\hline
\end{tabular}

tasa más baja en la Región Metropolitana además de inferior al promedio nacional ${ }^{16}$. Este hecho permite ratificar la hipótesis de este estudio: es factible disminuir la mortalidad por cáncer de mama en nuestro país. La disminución de cánceres avanzados (un desafío aún pendiente) y el aumento importante y creciente de cánceres incipientes permite pronosticar tasas de mortalidad aún más bajas a futuro.
Una debilidad se ha originado en algunas carencias de información derivadas de una recolección de datos en los aspectos diagnósticos a nivel de la población a veces incompleta, por insuficiente estructuración del sistema y por fallas humanas. En lo terapéutico, la información es más satisfactoria por desarrollarse en el ámbito hospitalario.

Se sostiene regularmente que una de las tasas más 
altas de mortalidad por cáncer de mama en Chile, si no la más alta, se observa en la Región Metropolitana ${ }^{12-14}$. Esto es efectivo pero, hasta ahora, nadie se había preocupado de conocer o destacar que existen grandes variaciones en la mortalidad por este cáncer al interior de la región, hecho de gran importancia no sólo epidemiológica sino también programática. Esta relevancia deriva de tratarse de grandes poblaciones y en consecuencia sus tasas de mortalidad tienden a ser más estables y confiables. Existe una experiencia chilena, justamente en cáncer de mama que lo ratifica ${ }^{32}$.

Por otra parte, la evaluación del programa nacional de cáncer de mama en la Región Metropolitana demuestra, a través de la experiencia del SSMSO, que se puede obtener un impacto sanitario positivo con reducción biológicamente significativa de la mortalidad por cáncer de mama en una población de gran tamaño. Es importante también que esto se haya logrado sin recurrir a financiamientos especiales sino más bien con un trabajo integrado ${ }^{16}$. En otras palabras, en este servicio de salud el programa ha sido eficiente ${ }^{33}$.

En nuestro medio no se ha debatido una estrategia de prevención primaria, o sea, intentando evitar que se produzca esta neoplasia. Esto se ha hecho en forma exitosa en base a una quimio-prevención antiestrogénica (Tamoxifeno, Raloxifeno) para pacientes de alto riesgo, en diferentes países desde fines de los 9020-24. Pueden contemplarse también otras estrategias preventivas: multivitamínicos, folatos, disminución de sobrepeso y obesidad especialmente en postmenopausia, control de tratamientos hormonales de reemplazo, fomento de multiparidad, de lactancia materna, de primer embarazo a menor edad, de ejercicio, control de stress, tratamiento de depresión, etc ${ }^{19}$.

Por otra parte, también deberían considerarse experiencias internacionales que enfatizan el diagnóstico precoz y tratamiento adecuado, según los niveles de desarrollo que tengan los países ${ }^{34-37}$.

Existe también en el SSMSO una buena opinión de sus usuarias. Ello probablemente promueve consultas por patología mamaria más precoces, lo que significa una menor proporción de cánceres avanzados y una mayor de incipientes, garantizando además el cumplimiento de este objetivo deseable en un programa de salud pública como es la prevención secundaria.

Creemos que la equidad del acceso a atención de salud se promueve, más allá de capacitaciones puntuales, con motivación y apoyo directo a médicos generales y matronas de parte de los especialistas hospitalarios en trabajo conjunto a nivel de los consultorios, tal como está ocurriendo en el SSMSO. En este sentido, es evidente por los datos entregados que el programa de cáncer de mama en este servicio de salud no presenta algunos de los problemas descritos en la literatura ${ }^{38}$.

Sin duda, se necesitan estudios adicionales que puedan explicar los buenos resultados del programa de cáncer de mama en el SSMSO, ya que aparentemente no existe una diferencia en la asignación de recursos ni del nivel de capacitación profesional por parte del Ministerio de Salud. Sin embargo, se puede proponer como factores diferenciadores la coordinación permanente entre especialistas y matronas de consultorios, lo cual significa motivación mutua y capacitación continua. Además, existe un gran compromiso profesional con excelente relación médico-paciente y tratamientos más oportunos, aunque esto no está medido en forma comparativa entre los distintos servicios metropolitanos. Sin embargo, debe señalarse que en el SSMSO no hay evidencia de que un aumento de procedimientos imagenológicos mamarios, apreciado sólo a partir del año 2007 en este servicio, y de exámenes de salud preventivos del adulto, pudieran explicar la mejoría observada de su mortalidad por cáncer de mama en la década actual.

Parece importante que la información entregada por los servicios de salud al nivel central, depurada de datos irrelevantes o no susceptibles de modificar por el ministerio, sea publicada periódicamente para su uso por cualquier grupo competente e interesado en su análisis, capaz de aportar al objetivo de disminuir la mortalidad por cáncer de mama, objetivo que en lo nacional no ha podido ser cumplido en una década y media de desarrollo.

Finalmente, debe insistirse que para avanzar verdaderamente en lo epidemiológico e incluso clínico en Chile, debería disponerse de un registro nacional de cáncer de mama o al menos registros regionales importantes: Santiago, Valparaíso y Bío Bío. Por supuesto, se deben aprovechar diversos esfuerzos desarrollados como registros locales de cáncer: Antofagasta, Valdivia (provincia), SSMSO y Concepción, además de experiencias desarrolladas tanto en el sector público ${ }^{39,40}$ como en el privado ${ }^{14}$.

\section{Referencias}

1. Phillips K-A, Glendon G, Knight JA. Putting the risk of breast cancer in perspective. New Eng J Med 1999; 340: 141-144.

2. Keating NL, Landrum MB, Guadagnoli E, Winer EP, Ayanian JZ. Surveillance Testing Among Survivors of Early-Stage Breast Cancer. J Clin Oncol 2007; 25: 1074-1081.

3. Fishler Y, Tarabeia J, Baron-Epel O, Buchena M, Liphshitz I, Epala A. A comparison of trends in incidence and 
mortality of breast cancer. Eur J Cancer Prev 2007; 16 : 36-42.

4. Kwan ML, Weltzien E, Kushi LH, Castillo A, Slattery ML, Caan BJ. Dietary Patterns and Breast Cancer Recurrence and Survival Among Women With Early-Stage Breast Cancer. J Clin Oncol 2009; 27: 919-926.

5. Phillip K-A, Osborne RH, Giles GG, Dite GS, Apicella C, Hopper JL, et al. Psychosocial Factors and Survival of Young Women with Breast Cancer: A PopulationBased Prospective Cohort Study. J Clin Oncol 2008; 26: 4666-4671.

6. Ismail J, Chen BE, Anderson WF, Rosemberg PS. Breast Cancer Mortality Trends in the United States According to Estrogen Receptor Status and Age at Diagnosis. J Clin Oncol 2007; 25: 1683-1690.

7. Sotiriou C, Pusztai L. Gene-Expression Signatures in Breast Cancer. N Eng J Med 2009; 360: 790-800.

8. Liu R, Wang X, Chen GY, Dalebo P, Gurney A, Noet $\mathrm{J}$, et al. The prognostic role of a gene signature from tumorigenic breast-cancer cells. N Eng J Med 2007; 356: 217-226.

9. Li X, Lewis MT, Huang J, Gutiérrez C, Osborne CK, Wen M-F, et al. Intrinsic Resistence of Tumorigenic Breast Cancer Cells To Chemotherapy. J Natl Cancer Inst 2008; 100: 672-679.

10. Pabst Y, Naranjo L, Power E. Cáncer de mama en Chile. Análisis de 1.358 casos. Rev Chil Cir 1980; 32: 304310.

11. Pabst Y. Epidemiología, etiopatogenia y evolución natural del cáncer de mama. En: Patología mamaria maligna. Ed. Y. Pabst, edit. Arancibia Hnos, 1992, Santiago, pp 13-25.

12. Peralta O. Cáncer de mama en Chile. Datos epidemiológicos. Rev Chil Obstet Ginecol 2002; 67: 439-445.

13. Peralta O. Diagnóstico, tratamiento y seguimiento de 1.036 mujeres con cáncer de mama en el Servicio Metropolitano Central, 2005. Citado por Acevedo JM (Ref. 14).

14. Acevedo JC, Rossat C, Gutiérrez V, Reyes JM. Cáncer de mama: experiencia del Centro Integral de la Mama Clínica Las Condes. Rev Med Clin Condes 2006; 17 : 248-255.

15. Jürgensen $M$, Chacón R, Baeza C, Riveros R. Cánceres de mama multifocales-multicéntricos ¿Son realmente de peor pronóstico? Rev Chil Cir 2009; 61: 125-130.

16. Serra I, Martínez R, Mimica X, Cavada G, Aguayo C. Cáncer de mama en Chile: un aporte clínico y epidemiológico en base a un registro poblacional metropolitano, 1.485 pacientes. Rev Chil Cir 2009; 61: 507-514.

17. Prieto M, Torres S. Situación epidemiológica del cáncer de mama en Chile 1994-2003. Rev Med Clin Condes 2006; 17: 142-148.

18. Medina E. Visión crítica del sistema de salud chileno. Rev Chil Salud Publ 1997; 1: 23-27.

19. Colditz GA. Epidemiology and Prevention of Breast Cancer. Cancer Epidem Biomar 2005; 14: 768-772.
20. Fisher B, Constantino JP, Wickerhan DL, Cecchini RS, Cronin WM, Robidoux A, et al. Tamoxifen for the prevention of breast cancer: current status of the National Surgical Adjuvant Breast and Bowel Project P-1 study. J Natl Cancer Inst 2005; 97: 1652-1662.

21. Cuzick J, Forbes JF, Sestak I, Cawthorne S, Hamed $\mathrm{H}$, Holli K, et al. Long-term results of tamoxifen prophylaxis for breast Cancer-96-month follow-up of the randomized IBIS-I trial. J Natl Cancer Inst 2007; 99: 272-282.

22. Vogel VG, Constantino JP, Wickerham DL, Cronin WM, Cecchini RS, Atkins JM, et al. Effects of tamoxifen vs raloxifen on the risk of developing invasive breast cancer and other disease outcomes: the NSABP Study of Tamoxifen and Raloxifen (STAR) P-2 trial. JAMA 2006; 295: 2727-2741.

23. Powles TJ, Asley S, Tidy A, Smith IE, Dowset M. Twenty-year follow-up of the Royal Marsden randomized, double-blinded tamoxifen breast cancer prevention trial. J Natl Cancer Inst 2007; 99: 283-290.

24. Veronesi U, Maissoneuve P, Rotmensz N, Costa A, Sacchini V, Travaglini R, et al. Italian Randomized Trial Among Women With Hysterectomy: Tamoxifen and Hormone-Dependent Breast Cancer in High-Risk Women. J Natl Cancer Inst 2003; 95: 160-165.

25. Acevedo JC, Baeza R, Fernández C, Ibarra A, Solé J, Solé A, Arriagada R. Cáncer de mama diagnosticado en un programa de detección precoz por mamografía (screening). Rev Chil Cancerología 1998; 8: 27-34.

26. Coldman A, Phillips N, Warren L, Kan L. Breast cancer mortality after screening mammography in British Columbia women. Int J Cancer 2007; 120: 1076-1080.

27. Tabar L, Yen M, Vital B, Chen H, Smith R, Duffy S. Mammography service screening and mortality in breast cancer patients: 20 years follow-up before and after introduction of screening. Lancet 2003; 361: 1405-1410.

28. Bolados A, Piérart J, Kascel S, Naveas R, Oliva L, Campos $\mathrm{S}$, y cols. Conocimientos generales sobre cáncer de mama en mujeres de Concepción. Rev Chil Cir 1996; 48: 397-401.

29. Piérart J, Burmeister R, Steinberg J, Muñoz C, Cid L, Cofré J, y cols. Autoexamen de mama. Rev Chil Cir 1994; 46: 522-527.

30. Weiss NS. Breast cancer mortality in relation to clinical breast examination and breast self-examination. Breast J 2003; 9 (suppl 2): S 86-S 89.

31. Montoya C. Equidad y eficiencia en el campo de la salud. En Seminario: Equidad y eficiencia en la toma de decisiones en salud. Ministerio de Salud, 1998, pp 93-112.

32. Piérart J, Lagos O, Cid L, Quijano P, Martínez A, Santander P. Mortalidad por cáncer de mama en la VIII Región de Chile. Rev Chil Cir 1996; 48: 608-618.

33. Cid C. Causas estructurales de los problemas de inequidad en el acceso a la salud en Chile. Rev Chil Salud Publ 2008; 12: 103-109. 
34. Anderson BO, Jakesz R. Breast Cancer Issues in Developing Countries: An Overview of the Breast Health Global Initiative. World J Surg 2008; 32: 2578-2585.

35. Vargas HI, Anderson BO, Chopra R, Lehman CD, Ibarra JA, Masood S, et al. Diagnosis of breast cancer in countries with limited resources. Breast J 2003; 9 Suppl 2: S60-S66.

36. Smith RA, Caleffi M, Albert US, Chen THH, Duffy SW, Franceschi D, et al. Breast cancer in limited-resource countries: early detection and access to care. Breast J 2006; 12 Suppl 1: S16-S26.

37. Shyyan R, Masood S, Badwe RA, Errico KM, Liberman $\mathrm{L}$, Ozmen V, et al. Breast cancer in limited-resource countries: diagnosis and pathology. Breast J 2006; 12
Suppl 1: 27-37.

38. Camacho J, Barriga C, Escobar P, Seleme N, Schwartz R. Costo beneficio del estudio de diseminación en estadios I y II del cáncer de mama. Rev Med Chile 2003; 131: 155-158.

39. Bolados A, Piérart J, Laferte W, Campos D, Herrera G. Centro de información sobre cáncer de mama. Propuesta de un sistema computarizado nacional. MAMANET. Rev Chil Cir 1996; 48: 637-641.

40. Aguayo C. Creación y explotación de un registro poblacional y hospitalario de cánceres del adulto en el Servicio Metropolitano Sur Oriente, 2001-2005. Tesis Magíster en Salud Pública, Escuela de Salud Pública, Universidad de Chile, 2007, 101 pág. 